\title{
A tribute to Ronald Melzack
}

\author{
Terence J Coderre PhD
}

$\mathrm{M}$ anuscripts in this issue and the Winter issue of Pain Research \& Management have been invited and published to honour Ronald Melzack for his seminal contributions to the study of pain and its treatment. This tribute follows Ronald Melzack's official retirement and his appointment as professor emeritus at McGill University, Montreal, Quebec. These issues present a series of manuscripts written by Ron's former students, fellows, collaborators and colleagues who have documented or reviewed original research, and provide a background on how Ron has influenced or inspired their research careers and ideas.

The manuscripts span a wide range of disciplines from spinal cord physiology and systems neuroscience to multidisciplinary pain clinics and palliative care. The diversity of the topics covered in these issues is a testament to the depth and breadth of Ron's own research interests. Ron's illustrious career will probably be remembered best for the gate control theory of pain that he coauthored with his long-time colleague and friend, Patrick Wall. The gate control theory has had a powerful impact on the pain field in terms of its influence on both scientific thinking and clinical practice. However, Ron will also be remembered for his leading contributions to various other areas of investigation, including the influence of culture on pain perception, the use of transcutaneous electrical nerve stimulation and psychological therapies for the treatment of chronic pain, the use of verbal descriptors as a means to measure and diagnose pain, ethical issues surrounding the administration of adequate doses of opioid medication for relief of chronic pain, and the significance of the pain experienced during labour, after burn injury and in patients with phantom limb pain.

In addition to the gate control theory of pain, Ron's legacy includes the following milestones in pain research. First, the development of the McGill Pain Questionnaire and the for- malin test, and gold standards for the measurement of pain in humans and for antinociceptive testing in animals. Second, the elaboration of the multidimensional nature of pain (sensory, affective and motivational) and its representation in the mammalian brain. Third, a theoretical description of the neuromatrix as a means of understanding the brain's ability to integrate incoming sensory inputs with past experience and with innate representations of the body schema and self. Furthermore, while Ron always credits John Bonica as the architect of the multidisciplinary pain clinic, and for his heroic efforts to establish pain research and management as an international priority, he should accept a significant degree of credit in this area. While not a clinician, Ron was the driving force behind the establishment of the McGill Pain Centre, one of the first multidisciplinary pain clinics in the world, and has been a leader in the worldwide push to recognize pain as a significant problem that needs immediate and lasting attention.

While Ron Melzack's name is synonymous with high quality research that is both meticulous and creative, it is Ron's empathy for those suffering from chronic pain, and his passion for understanding the true nature of the pathological processes that underlie such suffering, that has been an inspiration to all in the field. His passionate approach to understanding pain and his superb ability to communicate both what knowledge has been gained and the questions that still remain are perhaps two of Ron's most outstanding qualities. An enduring legacy is the enthusiasm for pain research and management that Ron has passed on to his students and colleagues, as can be witnessed in this tribute.

Thanks to the authors for their original contributions to this tribute; and on behalf of the authors and all those influenced by him, thanks to Ronald Melzack, for his multiple contributions to the pain field. 


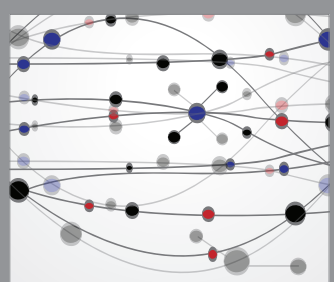

The Scientific World Journal
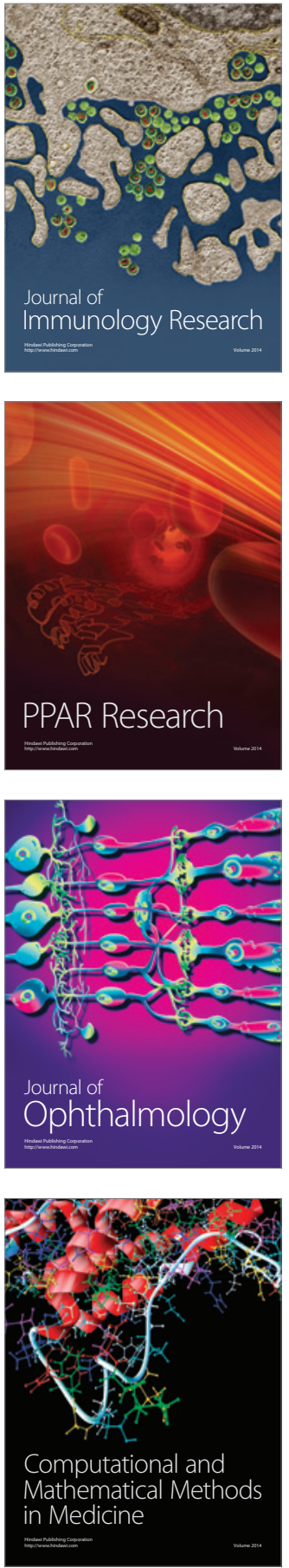

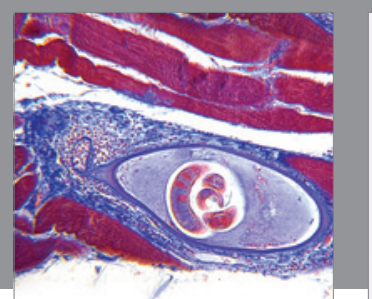

Gastroenterology Research and Practice

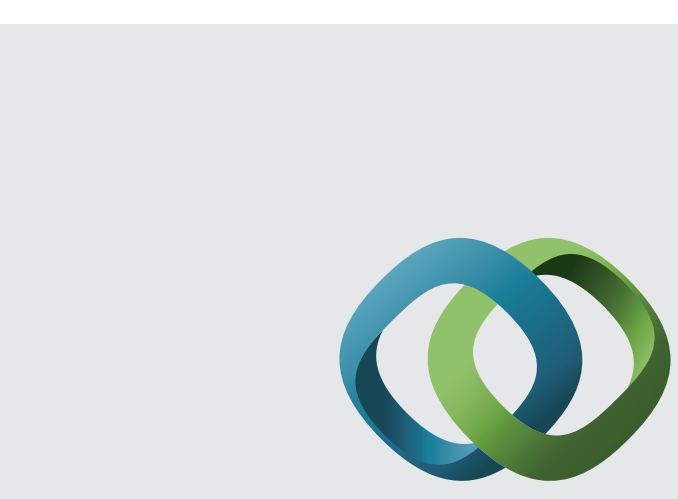

\section{Hindawi}

Submit your manuscripts at

http://www.hindawi.com
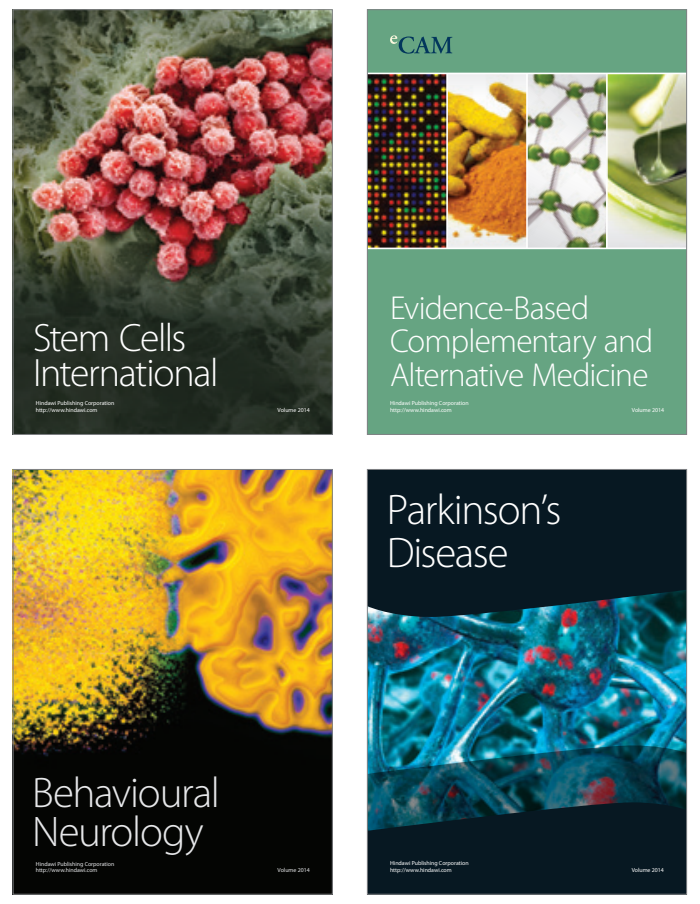
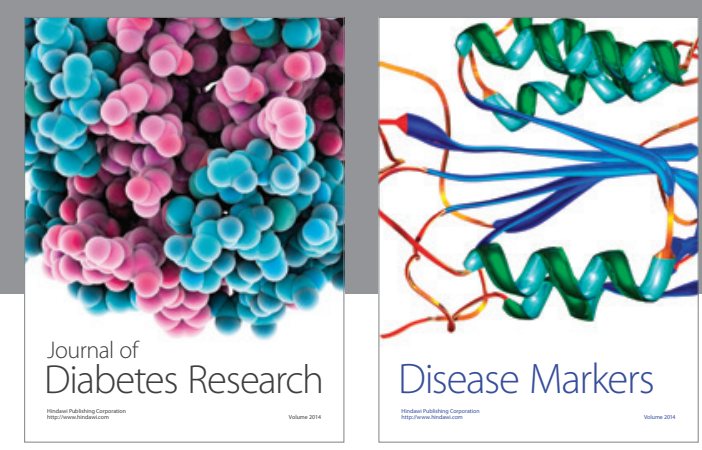

Disease Markers
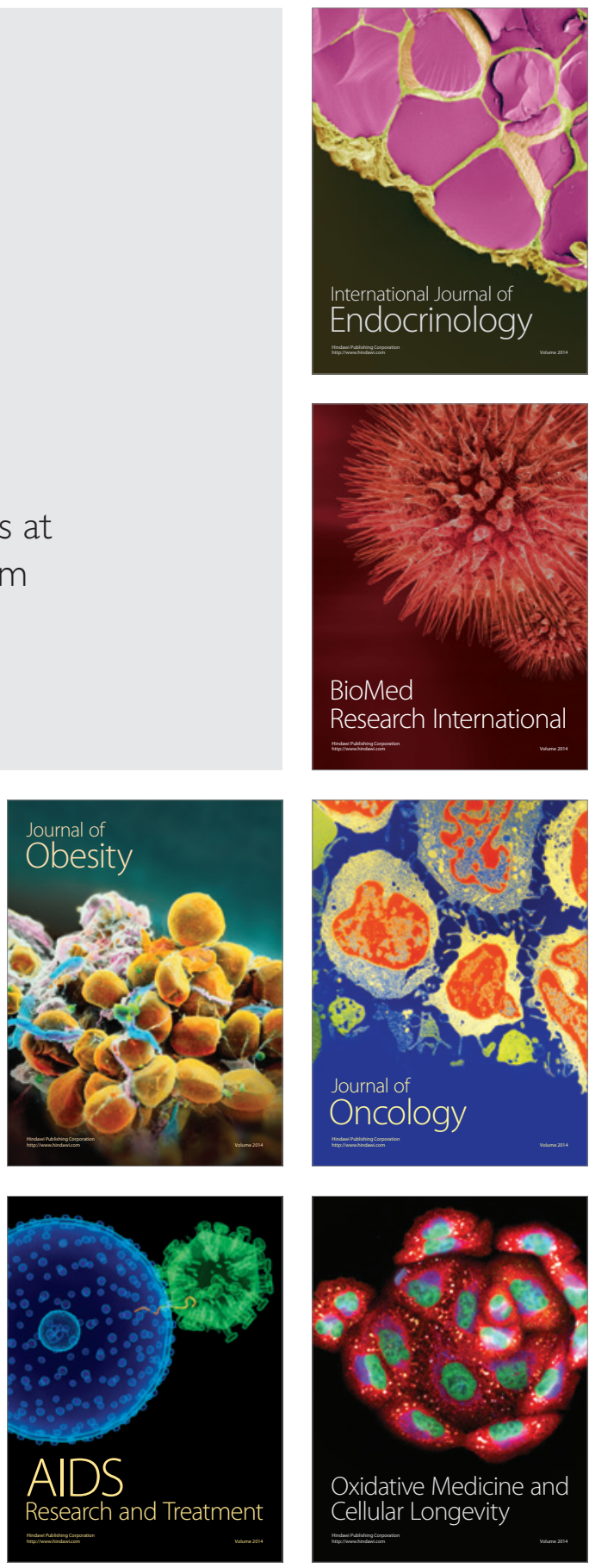